\title{
Répartition des Gammaridae épigés (Crustacés, Amphipodes) dans la Loire et les rivières de la Région Centre (France)
}

\author{
M. Chovet 1 \\ J.Y. Lécureuill
}

Mots clés : Répartition, Gammaridae, Echinogammarus berilloni, E. spinulicornis, Gammarus fossarum, G. p. pulex, G. roeseli, France, Région Centre, bassin de la Loire moyenne, bassin de la Seine moyenne.

Ce travail présente la répartition de 5 espèces de Gammaridae (Echinogammarus berilloni, E. spinulicornis, Gammarus fossarum, G. p. pulex, G. roeseli) dans la Région Centre qui correspond approximativement au bassin de la Loire moyenne et au sud du bassin de la Seine moyenne. Environ 300 stations ont été échantillonnées entre 1980 et 1990. G. fossarum n'est présente que dans l'Allier. L'espèce la plus répandue est $G$. p. pulex ; elle apparaît souvent en sympatrie avec $E$. berilloni. E. spinulicornis et $G$. roeseli sont deux espèces en extension dans la région. $E$. spinulicornis est nettement dominante à l'ouest, mais uniquement dans le bassin de la Loire, tandis que $G$. roeseli est plus abondante à l'est aussi bien dans le bassin de la Loire que dans celui de la Seine.

Distribution of epigean Gammaridae (Crustacea, Amphipoda) in the Loire river and in the streams of the Region Centre (France)

Keywords : Distribution, Gammaridae, Echinogammarus berilloni, E. spinulicornis, Gammarus fossarum, G. p. pulex, G. roeseli, France, Region Centre, middle Loire basin, middle Seine basin.

This work shows the distribution of five Gammaridae species (Echinogammarus berilloni, E. spinulicornis, Gammarus fossarum, G. p. pulex, G. roeseli) in the Region du Centre, an area including the Middle Loire Basin and the south of the Middle Seine Basin. About three hundred stations were sampled from 1980 to 1990. G. fossarum occurs only in the Allier river, G. $p$. pulex is the most common species, most often sympatric with $E$. berilloni, E. spinulicornis and $G$. roeseli are two species which have been spreading recently in this region. $E$. spinulicornis occurs mainly in the western part and only in the Loire basin, whereas $G$. roeseli occurs mainly in the eastern part both in the Seine and in the Loire basins.

\section{Introduction}

Si l'on excepte quelques informations anciennes (Pacaud 1944, 1952) ou concernant des régions limitrophes (Gras \& Maasen 1971, Pinkster \& Stock 1971, Goedmakers 1974), la répartition des Gammaridae (Crustacés, Amphipodes) épigés de la Région Centre était jusqu'à présent assez mal connue. Le but du présent travail est donc de faire un bilan de dix années de relevés faunistiques (de 1980 à 1990) effectués sur différents types de cours d'eau de cette région, relevés comprenant de nombreuses espèces de Gammares.

\footnotetext{
1.DIREN SEMA Centre, Cité administrative Coligny, 131 Faubourg Bannier, F-45042 Orléans Cedex.
}

\section{Caractéristiques de la Région Centre}

La Région Centre est une entité administrative correspondant à six départements. Cette Région est comprise entre le sud de la région parisienne et les derniers contreforts nord du Massif Central. Elle concerne deux grands bassins hydrographiques (Fig. 1) : celui de la Loire, le principal, et au nord celui de la Seine. C'est une région de faibles altitudes puisque celles-ci s'échelonnent de $450 \mathrm{~m}$ en bordure du Massif Central à $45 \mathrm{~m}$ à Tours. Les grandes cultures céréalières occupent le plateau de Beauce entre Chartres, Pithiviers et Orléans ; le Gâtinais, de l'Essonne à l'Yonne ; la pénéplaine du Berry autour de Bourges et Châteauroux. Les landes humides argilo-sableuses de Sologne s'étendent entre le Cher et la Loire. Ailleurs; les reliefs plus variés conservent leurs bocages, notamment dans le Sud. 
Le climat est tempéré par la proximité océanique, les influences atlantiques s'atténuent vers l'est de la région. Le régime hydrologique des rivières est de type pluvial, avec de hautes eaux en février et des étiages prononcés en août-septembre, en particulier dans le bassin de la Loire, alors que les nappes souterraines de la craie et des calcaires de Beauce soutiennent les débits du Loing, de l'Essonne et de l'Eure, affluents de la Seine. Les eaux des cours d'eau sont relativement minéralisées (40 à $100 \mathrm{mg}$ $\mathrm{Ca}^{++1-1}$ ), sauf celles qui sont issues de la bordure sud de la région (Allier, Loire, Cher, Vienne et Creuse notamment : 20 à $40 \mathrm{mg} \mathrm{Ca}^{++} \mathrm{l}^{-1}$ ) et celles qui drainent la Sologne ou le massif de la Forêt d'Orléans (10 à $20 \mathrm{mg} \mathrm{Ca}^{++1^{-1}}$ ).

\section{Origine des données}

La plus grande partie du matériel examiné provient de prélèvements effectués de 1980 à 1990 pour la détermination d'indices biotiques suivant la méthode de Tuffery \& Verneaux (1967) dans le cadre des programmes départementaux, régionaux et du RNB (Réseau National de Bassin) des Agences de l'Eau Loire-Bretagne et Seine-Normandie. Environ 300 sites ont été prospectés, la plupart une ou deux fois en cours d'étiage dans le cadre d'études des rivières, d'autres une ou plusieurs fois (6-8 fois) par an pour des suivis écologiques particuliers. Des prospections complémentaires ont été réalisées notamment hors de la Région Centre (Tableau I).

\section{Répartition des Gammaridae épigés dans la Région Centre}

Cinq espèces appartenant à deux genres ont été régulièrement rencontrées :

Echinogammarus berilloni Catta, 1978

Echinogammarus spinulicornis Pinkster \& Stock, 1971.

Gammarus roeseli Gervais, 1835

Gammarus pulex pulex (Linnaeus, 1758)

Gammarus fossarum Koch, 1835.

Echinogammarus berilloni et Gammarus pulex pulex sont les deux espèces les plus répandues. $G$. p. pulex est dominante ou est la représentante exclusive du groupe (Fig. 2) dans la partie amont des petits cours d'eau. Elle est également présente à l'aval d'importante arrivée d'eau fraîche en provenance de nappes souterraines comme dans l'Eure à l'amont immédiat de Chartres, dans la Cléry affluent rive droite du Loing ou dans 1'Yèvre à l'amont de sa confluence avec le Loir. Elle se rencontre également dans quelques petites rivières peu minéralisées (teneur en calcium inférieure à 20 $\mathrm{Ca}^{++1-1}$ ) comme les cours d'eau qui drainent strictement les sables et argile de Sologne (le Cosson et le Beuvron, mais non la Sauldre qui prend sa source en terrain calcaire) ou de la Forêt d'Orléans. Cette espèce est dominante sur de longs secteurs de grandes rivières du sud-est issues du Massif Central : le Cher moyen (à l'amont de Vierzon), la Loire Supérieure (à l'amont de la confluence avec l'Allier) et le début de la Loire Moyenne.

Echinogammarus berilloni est l'espèce la plus abondante dans le plus grand nombre de sites prospectés (Fig. 2). Elle est dominante dans la plupart des moyens et grands cours d'eau de la Région Centre, tandis que $G, p$. pulex occupe la partie amont des ruisseaux. $E$. berilloni régresse, et peut même disparaître, dans des secteurs fortement pollués (teneur en ammoniaque de 0,5 à $2 \mathrm{mg} \mathrm{l}^{-1}$ ou plus), comme on peut l'observer à l'aval d'agglomérations comme Châteauroux (Indre), Bourges (Yèvre), Montargis (Loing) (Fig. 5 et 6). Ce phénomène est également sensible à l'aval de Pithiviers (Essonne) et de Chartres (Eure) ; ces deux secteurs correspondent à des zones de sympatrie pour ces deux espèces.

G. fossarum est présente dans le Haut-Allier et quelques affluents de la Haute-Loire (Goedmakers 1974), elle a également été rencontrée dans l'Allier à l'aval de Moulins (Villeneuve-sur-Allier) où elle est dominante dans les secteurs courants (Fig. 3). Par contre, elle est absente de cette rivière au niveau de la confluence avec la Loire et dans tous les autres sites prospectés.

G. roeseli n'est présente que dans la partie Est de la Région Centre (bassin de Loire et de la Seine) (Fig. 3). Elle est également présente dans le Cher moyen de Saint Amand-Montrond à Vierzon (notamment dans les zones calmes), dans l'Yèvre entre Bourges et Vierzon et dans l'Auron, affluent de l'Yèvre, sur la quasi-totalité de son cours. Dans la Loire, elle est présente de l'amont de Decize (PortSt-Georges) jusqu'à l'aval d'Orléans (Muides), elle se rencontre dans la partie terminale des petits affluents situés en général dans le lit majeur de la Loire. Dans le bassin de la Seine elle est présente sur les deux tiers aval du Loing et dans la partie basse 


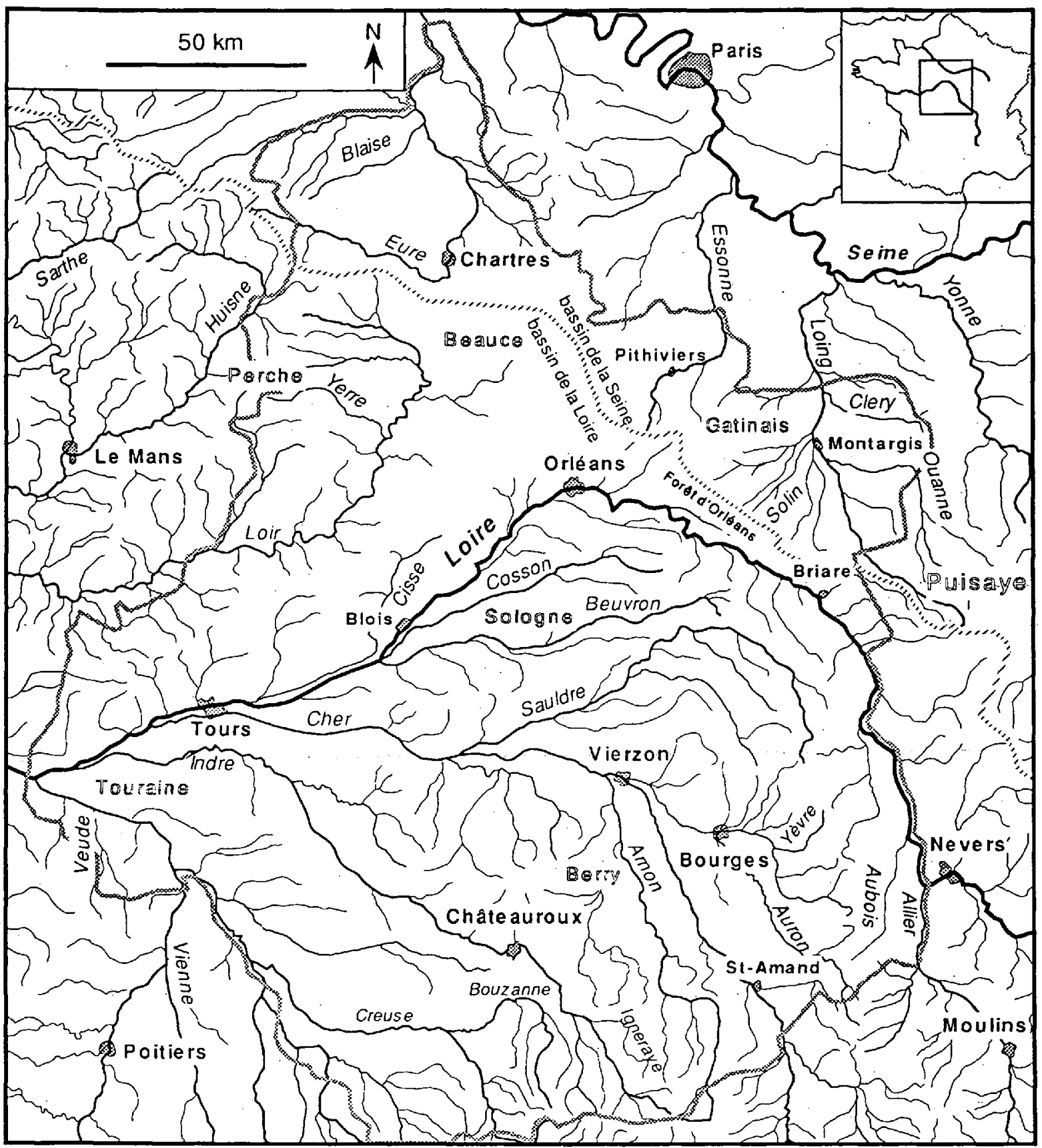

Fig. 1. Réseau hydrographique de la Région Centre (limite en grisé). Les grandes régions naturelles sont indiquées ainsi que le nom des principaux cours d'eau, de cours d'eau cités dans le texte (en italique) et le nom des principales villes (en caractères gras). La ligne épaisse en hachure qui va du nord-ouest au sud-est de la carte indique la limite des bassins de la Loire et de la Seine.

Fig. 1. Hydrographic lattice of the "Region Centre " (limits in grey). The major natural regions are indicated as well as the names of the principal rivers, the rivers cited in the text (in italics) and the names of the principal towns (in thick characters). The thick shaded line which goes from northwest to southeast on the map indicates the limits of the basins of the Loire and Seine. 


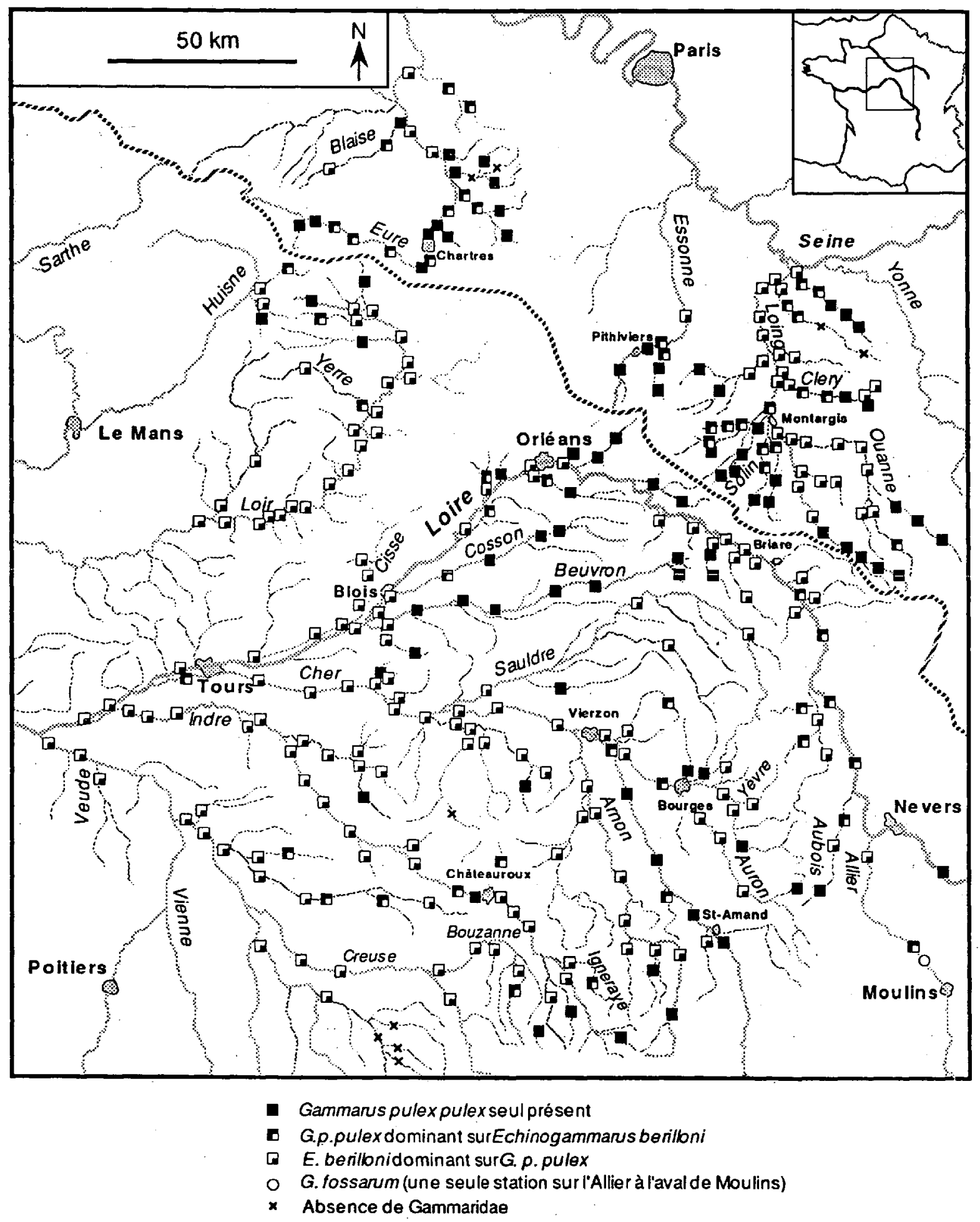

Fig. 2. Répartition géographique de Gammans puilex pulex et d'Echinogrammarus berilloni dans les cours d'eau de la Région Centre. Gammarus fossarum n'a été recueilli que dans une seule station sur l'Allier à l'aval de Moulins. Les X indiquent des stations où les Gammaridae sont absents: Carte de distribution établie à partir de divers types d'échantillonnage réalisés entre 1980 et 1990 (cf. également Tableau 1). La ligne épaisse en hachure qui va du nord-ouest au sud-est de la carte indique la limite des bassins de la Loire at de la Seine.

Fig. 2. Geographical distribution of Gammarus pulex and Echinogammarus berilloni in rivers of the "Region Centre ". Gammarus fossarum has been collected from only one station on the Allier downstream from Moulines. An X indicates a station where Gammaridae are absent. The distribution map is based on diverse types of samples taken from 1980 to 1990 (cf. likewise Table 1). The thick shaded line which goes from northwest to southeast on the map indicates the limits of the basins of the Loire and Seine. 
Tableau 1. Liste des cours d'eau inventoriés, nombre de stations sur chaque cours d'eau et fréquence des relevés. Affluents (I, II, III ou IV) ; I, affluent direct de la rivière principale (Loire ou Seine) ; II, affluent d'un affluent direct, etc... La dernière colonne indique le nombre de fois où les stations ont été visitées durant la décennie (1980-1990). "Annuelle " indique que certaines stations sont visitées chaque année. La position exacte des stations et leur description sont disponibles auprès des auteurs.

Table 1. List of rivers, number of stations on each river and frequency of sampling. Tributaries (I, II, III, or IV) : I, direct tributary of the principal river (Loire or Seine) ; II, tributary of direct tributary, etc... The last column gives the number of times on which the stations have been visited during the ten-year period (1980-1990). « Annual » indicates those stations visited each year. The exact position and description of the stations are available from the authors.

\begin{tabular}{|c|c|c|c|c|c|c|c|}
\hline $\begin{array}{l}\text { Rivières } \\
\text { Principales } \\
\end{array}$ & affluents (1) & affluents (II) & affluents (III) & affluents (IV) & $\begin{array}{c}\text { Nombre } \\
\text { de stations }\end{array}$ & $\begin{array}{c}\text { Nombre } \\
\text { des relevés }\end{array}$ & Observations \\
\hline \multirow[t]{40}{*}{ SEINE } & Essonne & & & & 4 & 1 & \\
\hline & & Rimarde & & & 3 & 1 & \\
\hline & Eure & & & & 11 & 1 à 2 & \\
\hline & & & & & 3 & 7 & annuelles \\
\hline & & Blaise & & & 2 & 1 à 4 & \\
\hline & & & & & 2 & 5 & annuelles \\
\hline & & Drouette & & & 2 & 1 à 7 & 1 annuelle \\
\hline & & & Guesle & & 1 & 1 & \\
\hline & & & Guéville & & 2 & 1 à 4 & \\
\hline & & Maltome & & & 1 & 1 & \\
\hline & & Roguenette & & & 1 & 1 & \\
\hline & & Ru de l'Ancie & Etang & & 1 & 2 & \\
\hline & & Vesgre & & & 2 & 1 à 7 & 1 annuelle \\
\hline & & Voise & & & 2 & 2 à 7 & 1 annuelle \\
\hline & & & Aunay & & 2 & 1 à 5 & 1 annuelle \\
\hline & & & Remarde & & 1 & 1 & \\
\hline & Loing & & & & 8 & 1 & \\
\hline & & & & & 7 & 5 à 11 & annuelles \\
\hline & & Aveyron & & & 3 & 1 & \\
\hline & & Betz & & & 2 & 1 & \\
\hline & & Bezonde & & & 2 & 6 à 9 & annuelles \\
\hline & & & & & 3 & 2 à 3 & \\
\hline & & & Huillard & & 2 & 1 à 2 & \\
\hline & & & Poterie & & 1 & 4 & \\
\hline & & Cléry & & & 8 & 1 à 2 & \\
\hline & & & Ru de Bougis & & 1 & 1 & \\
\hline & & Fusain & & & 3 & 2 à 5 & 1 annuelle \\
\hline & & & Maurepas & & 1 & 1 & \\
\hline & & Lunain & & & 4 & 1 & \\
\hline & & & & & 1 & 4 & \\
\hline & & Orvanie & & & 5 & 1 & \\
\hline & & & & & 1 & 5 & \\
\hline & & & Orval & & 1 & 1 & \\
\hline & & Ouanne & & & 8 & 1 à 7 & 2 annúelles \\
\hline & & & Branlin & & 2 & 1 & \\
\hline & & & & Agréau & 1 & 1 & \\
\hline & & Solin & & & 3 & 1 & \\
\hline & & & Treille & & 1 & 1 & \\
\hline & & Vernisson & & & 3 & 2 à 4 & \\
\hline & & & Puiseaux & & 4 & 1 à 2 & \\
\hline \multirow[t]{8}{*}{ LOIRE } & & & & & 19 & 1 à 3 & \\
\hline & & & & & 8 & 6 à $8 /$ an & annuelles \\
\hline & Allier & & & & 2 & 2 & \\
\hline & Aquiaulne & & & & 3 & 1 & \\
\hline & Ardoux & & & & 1 & 2 & \\
\hline & Aubois & & & & 4 & 1 & \\
\hline & Avenelle & & & & 1 & 1 & \\
\hline & Bec d'Able & & & & 1 & 1 & \\
\hline
\end{tabular}




\begin{tabular}{|c|c|c|c|c|c|c|c|}
\hline $\begin{array}{l}\text { Rivières } \\
\text { Principales }\end{array}$ & affluents (I) & affluents (II) & affluents (III) & affluents (IV) & $\begin{array}{c}\text { Nombre } \\
\text { de stations }\end{array}$ & $\begin{array}{c}\text { Nombre } \\
\text { des relevés }\end{array}$ & Observations \\
\hline & Beuvron & & & & 6 & 1 à 2 & \\
\hline & & Bièvre & & & 2 & 1 & \\
\hline & & Cosson & & & 6 & 1 à 2 & \\
\hline & & Gravotte & & & 1 & 2 & \\
\hline & & Surget & & & 1 & 2 & \\
\hline & Bionne & & & & 2 & 1 à 2 & \\
\hline & Bonnée & & & & 2 & 2 à 3 & \\
\hline & Cheuille & & & & 1 & 1 & \\
\hline & Cisse & & & & 5 & 1 à 2 & \\
\hline & & Brenne & & & 1 & 1 & \\
\hline & & Cisse Landai & & & 1 & 1 & \\
\hline & & Ru de la Fon & aine & & 1 & 1 & \\
\hline & Cher & & & & 11 & 1 à 3 & \\
\hline & & & & & 3 & 7 à 8 & \\
\hline & & Amon & & & 6 & 1 & \\
\hline & & & Portefeuille & & 2 & 1 & \\
\hline & & & Sinaise & & 1 & 1 & \\
\hline & & & Théols & & 1 & 1 & \\
\hline & & & & Varenne & 1 & 1 & \\
\hline & & Bavet & & & 1 & 1 & \\
\hline & & & Anguilleuse & & 1 & 1 & \\
\hline & & & & Beugnon & 1 & 1 & \\
\hline & & Fouzon & & & 4 & 1 à 3 & \\
\hline & & & Nahon & & 1 & 2 & \\
\hline & & & & Céphons & 2 & 1 & \\
\hline & & & Pozon & & 1 & 1 & \\
\hline & & & Renon & & 1 & 1 & \\
\hline & & Loubière & & & 1 & 1 & \\
\hline & & Ru de Renne & & & 1 & 1 & \\
\hline & & Sauldre & & & 3 & 1 à 4 & \\
\hline & & & Petite Sauldre & & 1 & 4 & \\
\hline & & & Rère & & 1 & 1 & \\
\hline & & Trian & & & 1 & 1 & \\
\hline & & Yèvre & & & 5 & 1 & \\
\hline & & & Airain & & 2 & 1 & \\
\hline & & & Auron & & 3 & 1 & \\
\hline & & & & Sagonin & 2 & 1 & \\
\hline & & & Barangeon & & 2 & 1 & \\
\hline & & & Colin & & 1 & 1 & \\
\hline & & & Langis & & 1 & 1 & \\
\hline & & & Louatier & & 1 & 1 & \\
\hline & & & Moulon & & 1 & 1 & \\
\hline & Indre & & & & 14 & 1 à 2 & \\
\hline & & & & & 2 & 4 à 6 & \\
\hline & & Couarde & & & 1 & 1 & \\
\hline & & Echandon & & & 1 & 1 & \\
\hline & & Igneraye & & & 2 & 1. à 3 & \\
\hline & & Indrois & & & 5 & 1 à 3 & \\
\hline & & & Olivet & & 1 & 1 & \\
\hline & & & Tourmente & & 1 & 2 & \\
\hline
\end{tabular}




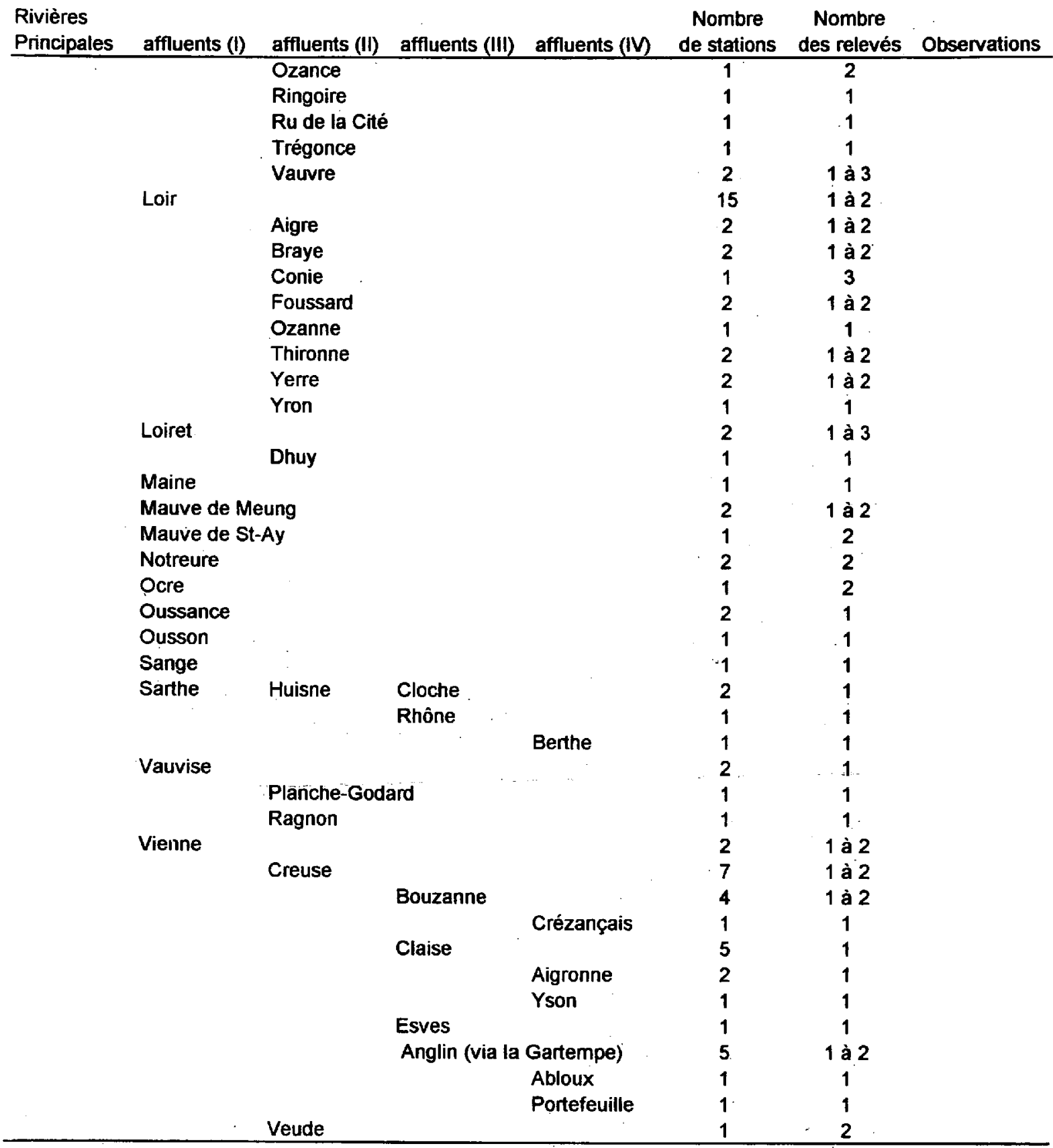

de ses affluents rive gauche ; elle remonte même la presque totalité du cours de certains d'entre eux tel le Solin, ruisseau recalibré qui n'est plus qu'une succession de biefs se déversant les uns dans les autres.

$C$. roeseli est sympatrique de $G$. $p$. pulex exclusivement dans le Cher moyen (sauf au voisinage de
Vierzon), dans le secteur terminal de la Loire Supérieure (de l'amont de Decize jusqu'à Nevers); et dans quelques petits cours d'eau comme le Solin (affluent du Loing, cf. supra) et l'Aubois amont, affluent de la Loire Moyenne près de Nevers, qui a la particularité d'être longé par le Canal du Berry reliant 
le Canal Latéral à la Loire au Cher. Ailleurs, elle est sympatrique d' $E$. berilloni qui est généralement dominante.

Echinogammarus spinulicornis est absente du bassin de la Seine (au moins des bassins du Loing, de l'Essonne amont et de l'Eure amont (Fig. 3). L'espèce est présente dans la Loire et ses affluents jusqu'à Blois. Elle se rencontre en effet dans le bassin du Cosson, du Beuvron, de la Cisse, du Cher, de l'Indre, de la Vienne et du Loir. Dans tous les sites prospectés elle est sympatrique d'E. berilloni. E. spinulicornis est parfois dominante ou subdominante dans quelques prélèvements de faune en particulier dans la Loire Moyenne dès l'aval de Tours, la Basse Loire et les secteurs du Cher, de l'Indre, de la Vienne, du Loir et de la Maine situés près de l'embouchure avec la Loire. Ces zones se situent à l'ouest d'un axe de pénétration majeure en eau continentale où les habitats principaux se caractérisent par une granulométrie fine (sables). Quelques petits cours d'eau offrent également des fonds limoneux et sableux apparemment très favorables à cette espèce que leur lit soit naturel (Bouzanne par exemple, Fig. 6) ou qu'il ait été aménagé par l'Homme (Igneraye, Veude). Par ailleurs, dans les rivières où E. spinulicorriis est présente, elle ne semble pas remonter aussi haut vers les sources qu' $E$. berilloni, comme c'est le cas dans le. Loir (Fig. 5). Enfin, à l'amont de Vierzon, à la fois dans le Cher et l'Yèvre, E. spinulicornis cohabite avec $G$. roeseli (Fig. 6). E. spinulicornis est même plus abondante qu' $E$. berilloni dans le Cher sur ce secteur.

La succession des espèces dans la Loire (et dans la partie aval de l'Allier) est la suivante (Fig. 4). $G$. $p$. pulex et $G$. roeseli se partagent les zones calmes et les annexes fluviales de la Loire supérieure avec des effectifs comparables, laissant libre le chenal soumis au courant. Dans l'Allier par contre $G$. fossarum est l'espèce dominante à l'aval de Moulins, $E$. berilloni lui succède en l'absence de $G$. roeseli. Après la confluence avec l'Allier, E. berilloni apparaît dans la Loire, elle envahit les zones des rives et les zones courantes et devient l'espèce dominante à l'aval de Briare où la densité des Gammaridae augmente considérablement ; $G$. roeseli disparaît entre Orléans et Blois, tandis que E. spinulicornis, apparue à Blois, est dominante ou subdominante de Tours à Nantes. Elle subsiste pratiquement seule dans le secteur influencé par les marées.

\section{Discussion}

Pacaud $(1944,1952)$ signalait que $G$. roeseli, espèce d'origine balkanique, absente du territoire français à la fin du XIX ${ }^{e}$ siècle, avait progressivement envahi les rivières françaises à partir de l'Alsace. En 1944 G. roeseli était déjà dominante dans la Seine aux environs de Paris et dans les grands cours d'eau de la partie orientale du bassin de la Seine tels que l'Yonne et la Marne. Pacaud (op. cit.) notait la progression de l'espèce dans le sud-est du bassin parisien, avec colonisation du Canal de Briare et du Loing à Montargis. L'auteur s'interrogeait sur les raisons pour lesquelles l'espèce n'avait pas encore investi le bassin de la Loire par le réseau de canaux.

Les observations récentes, ainsi que nos propres observations, montrent que l'extension de cette espèce vers le sud-est du bassin parisien s'est poursuivie jusqu'au bassin de la Loire, sans doute par les canaux parallèles au Loing par Briare, à l'Yonne par Decize ou par le Canal du Centre reliant la Saône à la Loire par Digoin. A partir du fleuve ou de son canal latéral, l'espèce a gagné le bassin du Cher par les branches du Canal du Berry parallèles à des rivières telles que l'Aubois, l'Auron et l'Yèvre. Cependant elle n'a pas encore colonisé le Cher à l'ouest de Vierzon malgré des conditions favorables représentées par la continuité du Canal et les aménagements de la rivière elle-même jusqu'à Tours. Siegismund (1988) indique que $G$. roeseli est une espèce tolérante à la pollution.

Dans le bassin du Rhône $G$. roeseli a continué à progresser vers le sud accompagnée de $E$. berilloni (Roux et al. 1980, Jazdzewski \& Roux 1988).

E. spinulicornis n'est pas signalée dans le bassin de la Loire aux environs de Nantes (Loire et affluents tels que l'Erdre et la Sèvre nantaise) par Gras \& Maasen (1971). Cette espèce présente en Aquitaine avait pour limite septentrionale la rivière Vendée (Gras \& Maasen op. cit.). Nos observations montrent que cette espèce a envahi le bassin de la Loire au cours des années 70 , remontant le fleuve jusqu'à Blois.

$E$. berilloni, espèce du domaine atlantique a étendu récemment son aire de répartition vers le Nord et l'Est. Nos observations montrent que la limite d'extension de cette espèce au sud-est a peu varié depuis les observations de Pacaud (1952). Elle a cependant progressé le long de la Loire depuis 


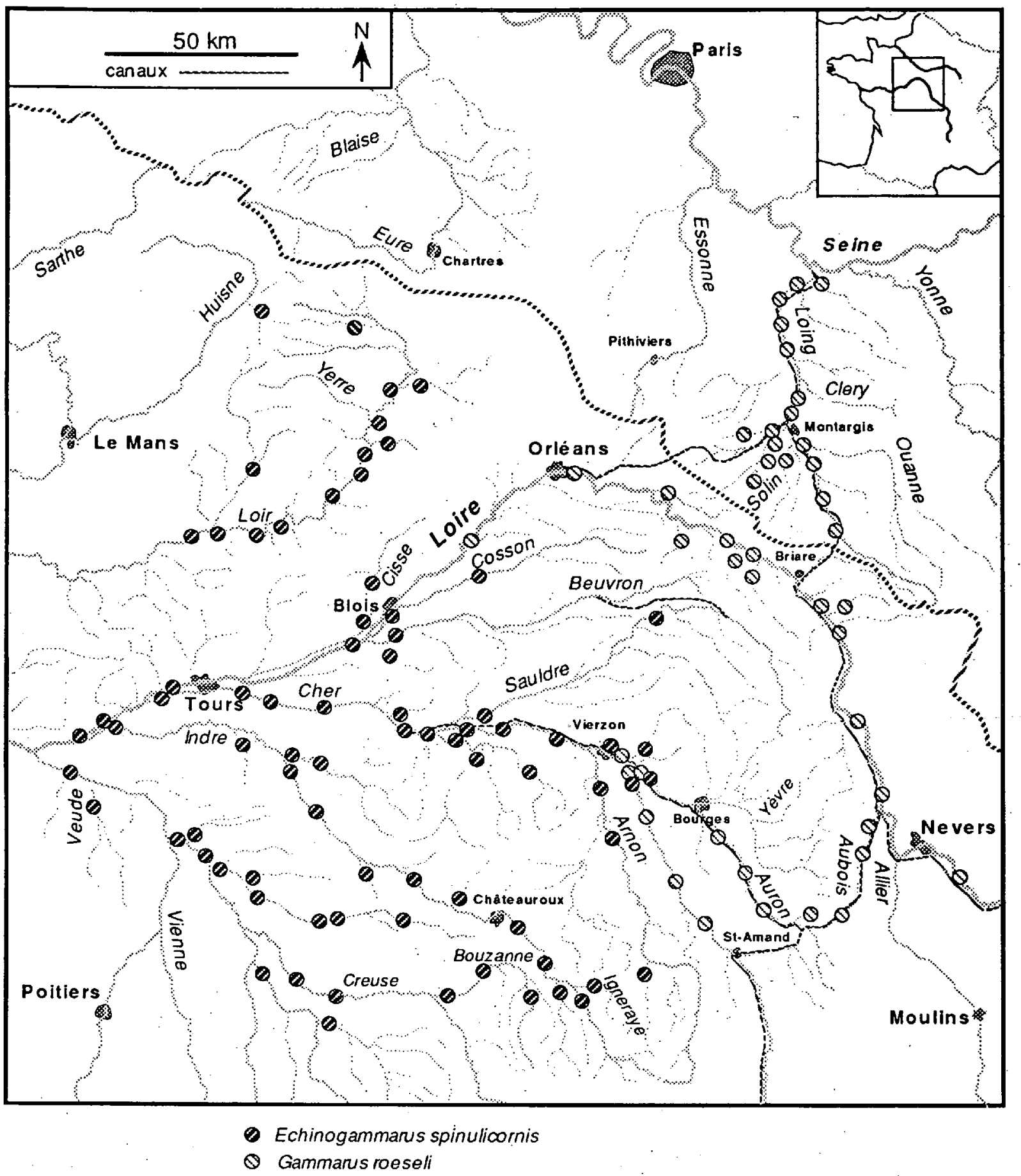

Fig. 3. Répartition géographique de Gammarus roeseli et Echinogammarus spinulicornis dans les cours d'eau de la Région Centre. La position des canaux reliant divers hydrosystèmes est également indiquée. Carte de distribution établie à partir de divers types d'échantillonnage réalisés entre 1980 et 1990 (cf. également Tableau 1). La ligne épaisse en hachure qui va du nord-ouest au sud-est de la carte indique la limite des bassins de la Loire et de la Seine.

Fig. 3. Geographical distribution of Gammarus roeseli and Echinogammarus spinulicornis in rivers of the "Region Centre ". The position of the canals connecting various hydrosystems are clearly indicated. The distribution map is based on diverse types of samples taken from 1980 to 1990 (cf. likewise Tabie 1). The thick shaded line which goes from northwest to southeast on the map indicates the limits of the basins of the Loire and Seine. 


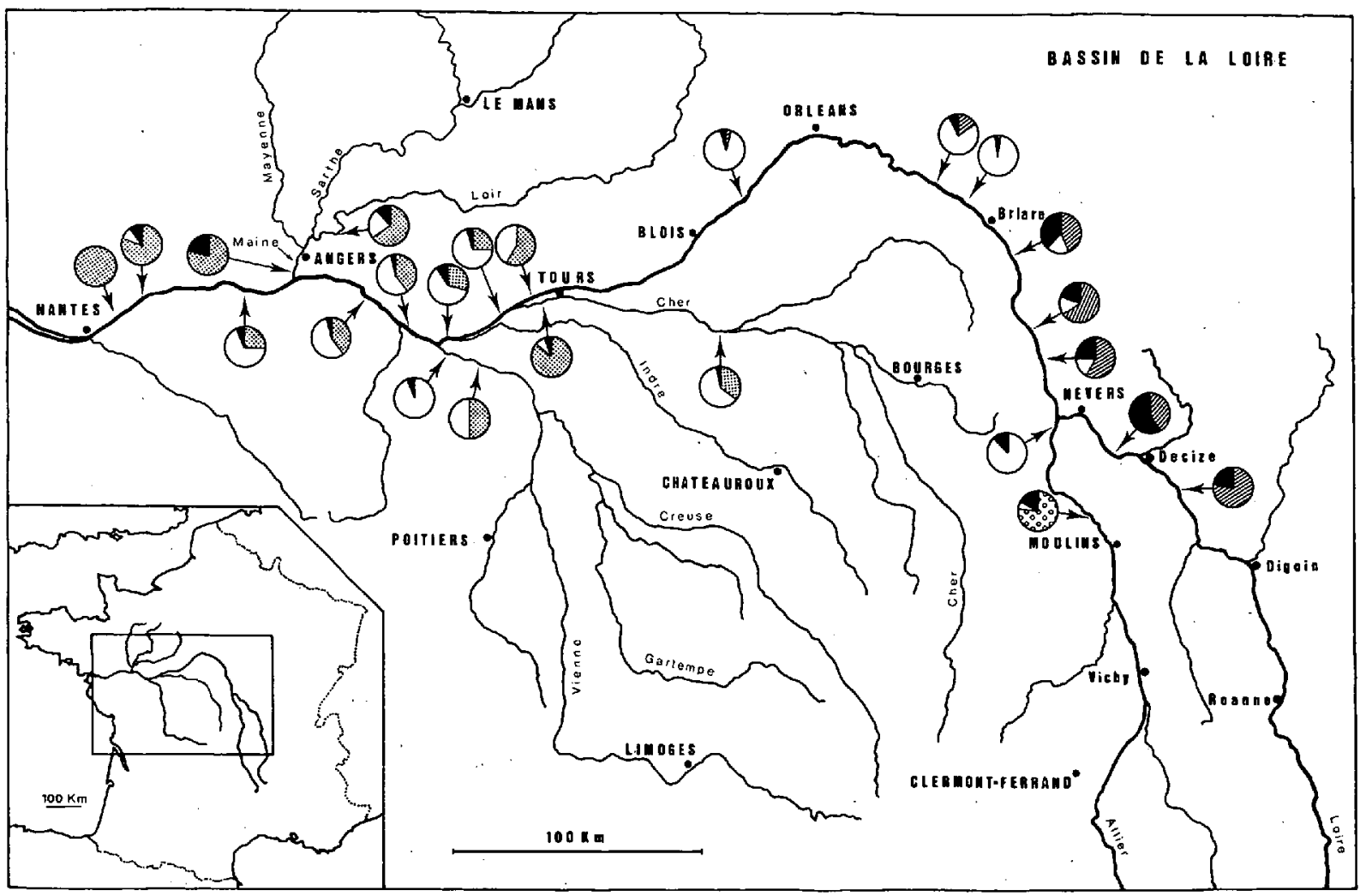

Fig. 4. Distribution des Gammaridés le long de la Loire et de ses principaux affluents. Amont d'Orléans : inventaires d'avril et juin 1983.

Aval d'Orléans : inventaires de mai-juin 1982 et début juillet 1983.

En noir : $G$. pulex pulex; en blanc : $E$. berilloni ; lignes obliques : $G$. roeseli; en grisé : $E$. spinulicornis.

L'angle de chaque secteur est proportionnel à l'abondance relative de chaque espèce.

Fig. 4. Distribution of gammarids along the Loire and its chief tributaries.

Upstream from Orléans : data from April to June 1983.

Downstream from Orléans : data from May-June 1982 to start of July 1983.

In black : $G$. pulex pulex; in white : $E$. berilloni ; oblique lines : $G$. roeseli ; in grey : $E$. spinulicornis.

The angle of each sector is proportional to the relative abundance of each species.

Briare pour atteindre le cours inférieur de l'Allier. Par contre si l'espèce est bien présente dans quelques affluents du Cher moyen vers Saint AmandMontrond (Loubière, Trian), elle semble ne pas pouvoir s'installer durablement dans le Cher lui-même.

G. p. pulex est présente partout, mais inégalement abondante. Goedmakers (1974) a signalé son absence dans certains cours d'eau du Massif Central (cf. la partie amont du bassin de. l'Anglin affluent de la Creuse, Fig. 2). La présence de $G$. p. pulex, comme celle de G. fossarum, ainsi que $G$. wautieri Roux 1967 et $G$. orinos Pinkster
\& Scholl 1984 dans les eaux continentales françaises est relativement ancienne (avant les glaciations pleistocène). G. p. pulex et $E$. berilloni sont nettement dominantes dans la Région Centre. G. p. pulex est actuellement en compétition avec $E$. berilloni, même si cette compétition d'après Goedmakers (1981) et Pinkster (comm. pers.) est limitée grâce à un partage de temps, le cycle biologique des deux espèces étant décalé.

G. fossarum, espèce abondante dans les Alpes, est plus rare dans les cours d'eau du Massif Central (Goedmakers 1974). Pour la région concernée par 


$$
\text { LE LOING }
$$

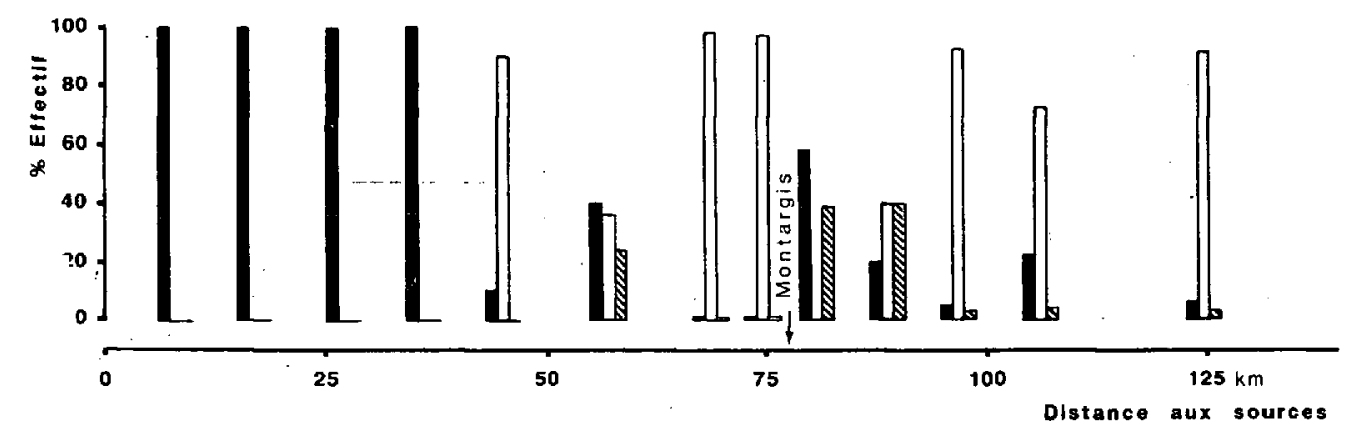

L'INDRE

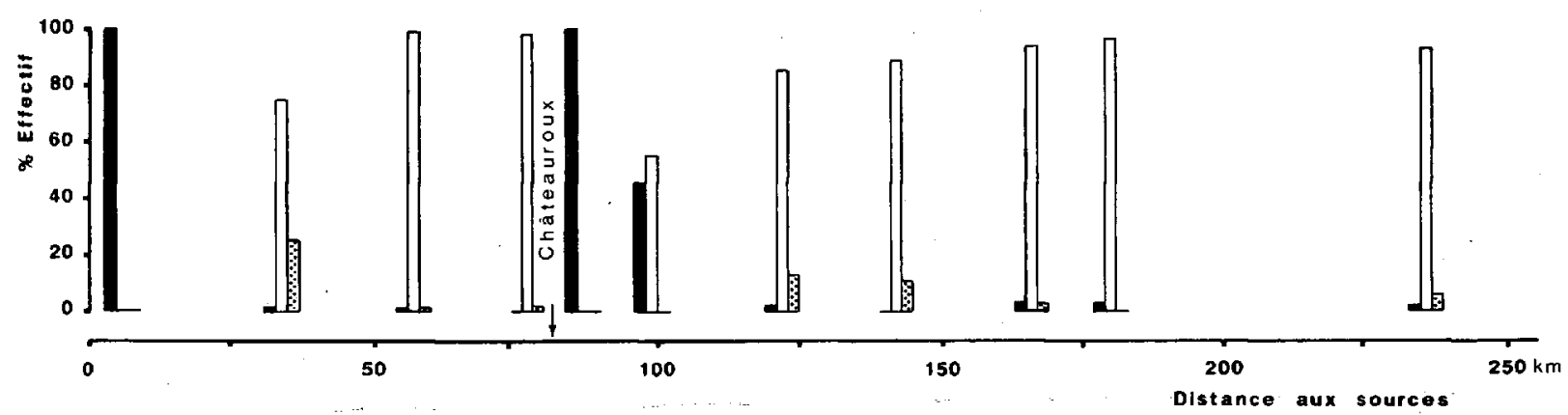

LE LOIR

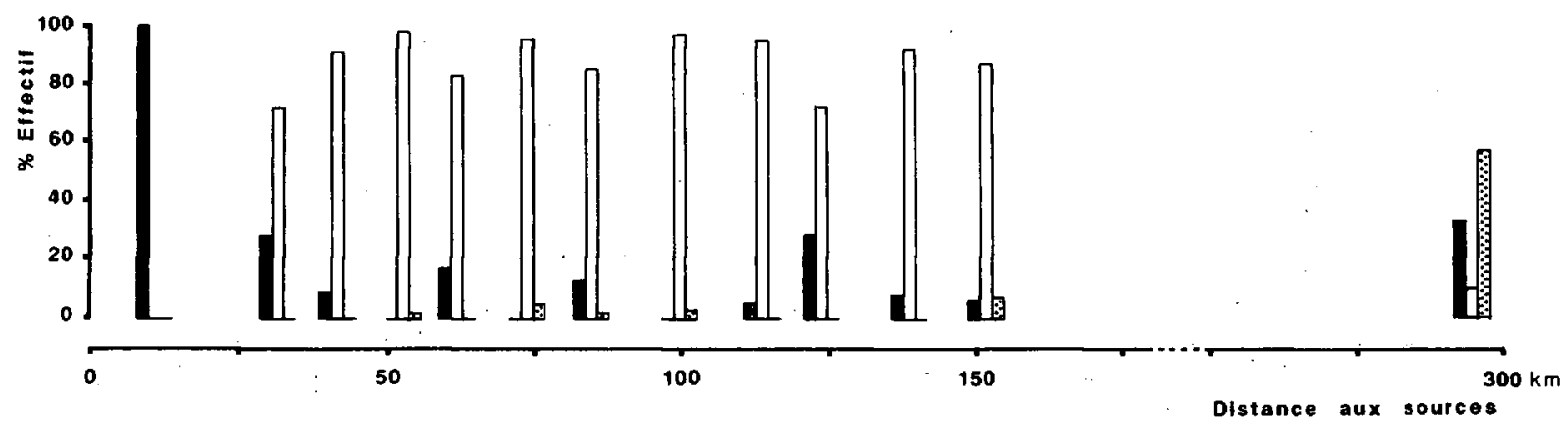

Fig. 5. Distribution longitudinale des Gammaridés de quelques rivières : le Loing (août 1988) ; l'Indre (juillet 1984) ; le Loir (août 1986, sauf la dernière station en juillet 1983).

En noir : $G$. pulex pulex; en blanc : $E$. berilloni; lignes obliques : $G$, roeseli ; en pointillé : E. spinulicornis.

Fig. 5. Longitudinal distribution of gammarids in some rivers : the Loing (August 1988); the Indre (July 1984) ; the Loir (August 1986, apart from the last station in July 1983).

In black : G. pulex pulex; in white : $E$. berilloni; oblique lines : G. roeseli ; stippled : E. spinulicornis. 
LE CHER

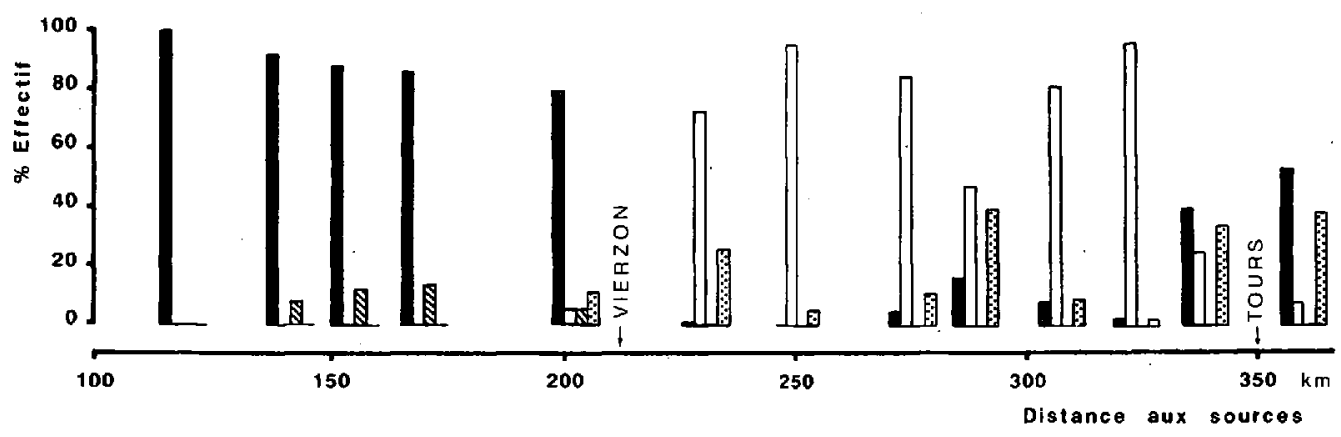

LA. LOIRE

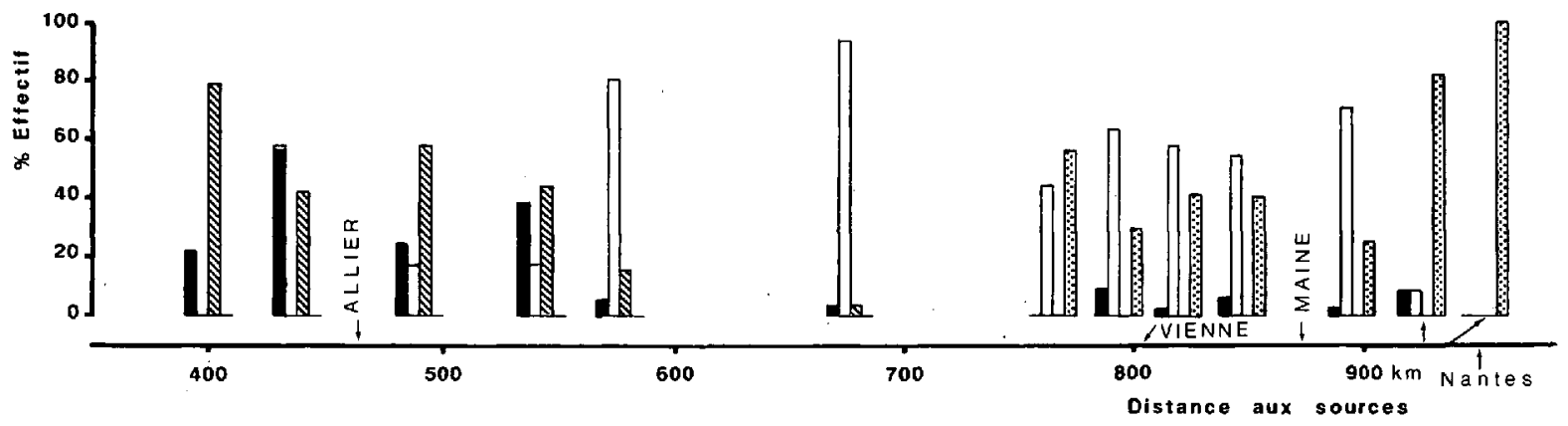

L' YEVRE

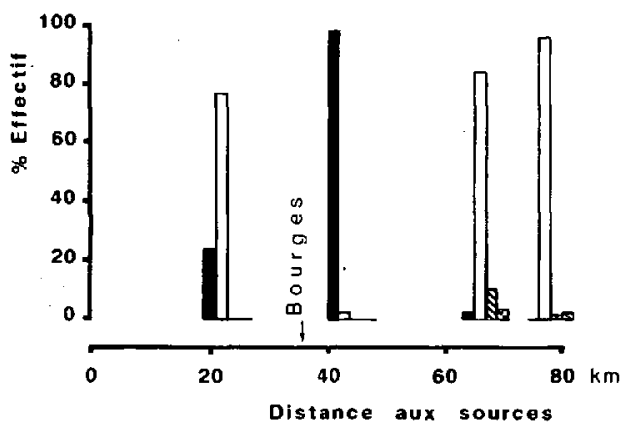

LA BOUZANNE

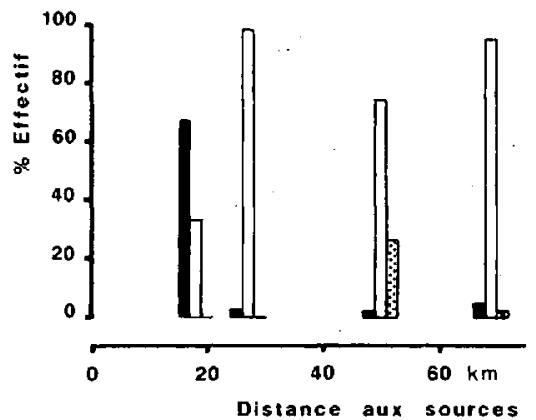

Fig. 6. Distribution longitudinale des Gammaridés de quelques rivières : le Cher (octobre 1988) ; la Loire (juin 1982 et juillet 1983) ; l'Yèvre (octobre 1980) ; la Bouzanne (juillet 1985).

En noir : $G$. pulex pulex; en blanc : $E$. berilloni ; lignes obliques : $G$. roeseli ; en pointillé : $E$. spinulicornis.

Fig. 6. Longitudinal distribution of gammarids in some rivers : the Cher (October 1988); the Loire (June 1982 and July 1983); the Yèvre (October 1980); the Bouzanne (July 1985).

In black : $G$. pulex pulex; in white : E. berilloni ; oblique lines : G. roeseli ; stippled : E. spinulicornis. 
cette étude elle n'est signalée que dans la moyenne vallée de l'Allier.

Cette étude, reposant sur un jeu de données hétérogènes, ne nous permet pas de faire la part des facteurs biotiques et abiotiques dans l'actuelle répartition des 5 espèces de Gammaridae de la Région Centre. Parmi les facteurs abiotiques, la température, le courant et la teneur en calcium sont très certainement des facteurs importants comme le montrent les travaux de divers auteurs dont Roux (1971), Barbier \& Champ (1974) et Roger \& Faessel (1989) pour la température, Vincent (1971) et Pinkster (1973) pour la teneur en calcium, Gras \& Maasen (1971) et Roux et al (1980) pour le courant. Parmi les facteurs biotiques susceptibles d'intervenir, outre les phénomènes possibles de compétition puisque très souvent il y a sympatrie, on peut également penser que chez des espèces comme $G$. roeseli, $E$. berilloni ou $E$. spinulicornis, mais cela est également vrai pour une espèce considérée comme plus « sédentaire " telle que $G$. fossarum, il existe une incontestable dynamique populationnelle conduisant ces espèces à coloniser sans cesse de nouveaux cours d'eau (Goedmakers 1981, Scheepmaker 1987).

En définitive cette étude confirme la présence de 3 espèces déjà connues dans le bassin de la Seine. Dans le bassin de la Loire elle révèle la présence de deux nouvelles espèces $G$. roeseli venue probablement par les réseaux de canaux du nord-est et $E$. spinulicornis, jusque là cantonnée aux Charentes et à la Vendée, qui a envahi la Loire et ses affluents de l'ouest jusqu'à Blois. Parmi les 3 espèces « autochtones », G. fossarum est localisée sur l'Allier uniquement, $E$. berilloni est la plus abondante sur la plupart des sites, $G$. $p$. pulex est plus répandue que la précédente mais ses effectifs sont souvent peu abondants sauf dans les parties amont des affluents et à l'aval des secteurs pollués où elle se révèle plus résistante.

Il est possible, outre des facteurs intrinsèques, que l'expansion de $E$. berilloni et $G$. roeseli ait été accélérée par les phénomènes de recalibrage et de régularisation des cours d'eau supprimant les macrophytes.

\section{Remerciements}

Les auteurs remercient Mme C. Roux (Lyon) pour l'aide apportée à l'identification des Gammares et M. H. Tachet (Lyon) pour ses remarques et suggestions lors de la rédaction du manuscrit.
Travaux cités

Barbier B. \& Champ P. 1974. - Résistance des.Gammares de la Seine aux élévations de températures. Bull.fr. Pisc., 225 : $67-71$.

Goedmakers A. 1974. - Les Gammaridae (Crustacés, Amphipodes) du Massif Central: Bull. zool. Mus. Univ. Amsterdam, 3 : 211-219.

Goedmakers A. 1981. - Population dynamics of three gammarid species (Crustacea, Amphipoda) in a French chalk stream. Part III. Migration. Bijdr Dierk, 51 : 145-180.

Gras J:M. \& Maasen A.M. 1971. - Les Gammaridés des eaux continentales et saumâtres du sud-est de la région armoricaine et du nord du bassin d'Aquitaine. Bijdr. Dierk. 41 : 52-60.

Jazdzewski K. \& Roux A.L. 1988. - Biogéographie de Gammarus roeseli Gervais en Europe, en particulier répartition en France et en Pologne. In : Proceedings of the VIth International Colloquium on Amphipod Crustaceans, Ambleteuse, France, 28 june - 3 july 1985. Crustaceana, Suppl, 13 : 272-277.

Karaman G.S. \& Pinkster S. 1977. - Freshwater Gammarus species from Europe, North Africa and adjacent regions of Asia (Crustacea-Amphipoda). Part 1. Gammarus pulex-group and related species. Bijdr. Dierk. $47: 1-97$.

Pacaud A. 1944. - Sur les Amphipodes gammariens des eaux superficielles dans la région centrale du Bassin Parisien. Bull. Soc. zool. France, $69: 33-46$.

Pacaud A. 1952. - Nouvelle revue de la distribution géographique des Gammares dans les eaux continentales françaises. C.R. Soc. biogéogr., 252-253: 95-111.

Pinkster S. 1973. - The Echinogammarus berilloni-group, a number of predominantly iberian amphipod species (Crustacea). Bijdr. Dierk. 43 ; 1-39.

Pinkster S. \& Scholl A. 1984. - Gammarus orinos n. sp. from the Massif Central (France) : its genetic and morphological distinction from Gammarus ibericus Margalef, 1951 (Crustacea, Amphipoda). Bijdr. Dierk. 54 : 139-146.

Pinkster S. \& Stock J.H. 1971. - Sur deux Echinogammarus limniques nouveaux du bassin d'Aquitaine, Bijdr. Dierk. $41^{\text {: }}$ $37-51$.

Roger M.C. \& Faessel B. 1989. - Effets de l'échauffement artificiel de l'eau du Rhône sur le développement et la production des Gammaridae. Hydroécol. Appl. 1/2 : 53-83.

Roux A.L. 1971. - Les gammares du groupe pulex. Essai de systématique biologique. Quelques caractéristiques écologiques et physiologiques. Arch. Zool. exp. gen. $112: 471-503$.

Roux C., Roux A.L. \& Opdam Y. 1980. - Répartition écologique et métabolisme respiratoire de Gammarus roeseli Gervais, 1835. Crustaceana, Suppl. $6: 148-159$.

Scheepmaker M: 1987. - Morphological and genetic differentiation of Gammarus stupendus Pinkster, 1983 in the Massif de la Sainte Baume, France. Bijdr. Dierk. 57 : 1-18.

Siegismund H.R. 1988. - Genetic differentiation in populations of the freshwater amphipods Gammarus roeseli and $G$. fossarum. Hereditas, $109: 269-276$.

Tuffery G. \& Verneaux J. 1967. - Méthode de détermination de la qualité biologique des eaux courantes. Trav. Div. Qual. Eaux P. Pisc., C.T.G.R.E.F., 23 p. 